\title{
Accounting for Financial Investments in View of International and Local Financial Reporting Standards
}

\author{
Ayad Jumaah Khalaf*، Omar Mohammed Arkad \\ University Headquarters‘ University of Anbar‘ Iraq \\ *ayadjumaa97@gmail.com
}

\begin{abstract}
:
The transformations in the business environment and the opening of markets have led to increased interest in financial investments to revive the national economy and with the emergence of international business and multinational companies، accounting work has become more extensive and complex ‘ which requires improving local accounting standards and in line with international requirements * where the research problem is that Iraq is one of the countries It seeks to use accounting that is largely consistent with the international financial reporting standards through its keenness to apply the unified accounting system and accounting rules and that studying financial investments in the light of both international financial reporting standards and Iraqi accounting regulations and knowing and identifying similarities and differences between them can improve the process of increasing Harmony and increased compatibility Where the importance of research lies in improving the unified accounting system and the Iraqi accounting rules through which financial investments are accounted for and shown in financial statements and communicated to users of financial reports in a way that reflects on the enhancement of investment decisions، and with this the aim of the research is to identify financial investments in the light of each of the reporting criteria International and local financial and accounting treatments in addition to conducting a comparative analytical study to find out the similarities and differences between them and proposing the necessary basis and procedures to help increase harmony and consensus' as the two researchers used the theoretical side and the comparative analytical method based on the sources and references as well as the international and local financial reporting standards related to the research topic Also، tables and illustrations were used، many results were reached' the most important of which is a wide gap between international and domestic financial reporting standards in relation to accounting for financial investments. Financial statements a and among the recommendations of the researchers is the necessity to issue a separate accounting rule for financial investments and another rule for investments in real estater as well as an accounting base for investment in associates and joint ventures in order to classify financial investments according to what came in the international financial reporting standards.
\end{abstract}

Keywords: $\quad$ Financial Investments; Financial Instruments IFRS 9; Unified Accounting System; Iraqi Accounting Base No. (14).

Crossref doi https://doi.org/10.51345/.v31i2.227.g181 


\section{المحاسبة عن الاستثلارات المالية في ضوء معايير الإبلاغ المالي الدولية والمحلية اياد جمعه خلف *، عمر محمد عر كد رئاسة الجامعة، جامعة الأنبار، العراق \\ * ayadjumaa97@gmail.com}

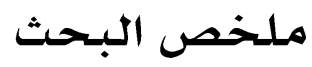
أدت التحو لات في بيئة الاعحال وانفتاح الاسواق الى زيادة الاهتحام بالاستثارات المالية ومع ظهور الاعحال الدولية والشركات متعددة الجنسيات اصبحت اعحال المحاسبة اكثر اتساعا وتعقيدا مما يتطلب تحسين معايير المحاسبة المحلية وبها يتلاءم مع المتطلبات الدولية، حيث تتمثل مشكلة البحث في كون العراق من الدول التي تسعى الى استخدام محاسبة تتلاءم الى حد كبير مع معايير الابلاغ المالي الدولية من خلال حرصها على تطبيق النظام المحاسبي الموحد والقواعد المحاسبية وان دراسة الاستثلارات المالية في ضوء كل من معايير الابلاغ الملالي الدولية واللوائح المحاسبية العراقية وتحديد اوجه التشابه والاختلاف بينها يمكن ان يحسن من عملية الانسجام والتوافق، حيث تكمن اهمية البحث في تحسين النظام المحاسبي الموحد والقواعد المحاسبية العر اقية، وبهذا هدف البحث المى التعرف على الاستثلارات المالية في ضوء كل من معايير الابلاغ المالي الدولية والمحلية وبيان المعالجات المحاسبية بالإضافة الى اجراء دراسة تحليلية مقارنة لمعرفة اوجه التشابه والاختلاف بينها واقتراح الاسس والاجراءات اللازمة بحا يساعد في زيادة الانسجام والتوافق، حيث استخدم الباحثان الجانب النظري والاسلوب التحليلي المقارن بالاعتحاد على المصادر والمراجع فضلا عن معايير الابلاغ الملالي الدولية والمحلية المتعلقة بموضوع البحث، كما تم استخدام الجداول والاشكال الايضاحية، تم التوصل الى العديد من النتائج اهمها وجود فجوة واسعة بين معايير الابلاغ الملالي الدولية والمحلية في ما يتعلق بالمحاسبة عن الاستثلارات المالية فمثلا هناك اختلاف من حيث المصطلحات الواردة فيها والنطاق والمدف ومن حيث تصنيف الاستثلارات المالية واثباتها والافصاح عنها في القوائم المالية، ومن توصيات الباحثان ضرورة اصدار قاعدة محاسبية منفصله للاستثحارات المالية وقاعدة اخرى الاستثلارات في العقارات وكذلك قاعدة محاسبية للاستثلار في الشر كات الزميلة والمشاريع المثتركة من اجل تصنيف الاستثلارات المالية وفق ما جاءت في معايير الابلاغ المالي الدولية.

الكلمات المفتاحية: الاستثمهارات المالية، الادوات المالية، النظام المحاسبي الموحد، القاعدة المحاسبية العراقية رقم 14. Crossref doi https://doi.org/10.51345/.v31i2.227.g181 
المقلدمة:

التحولات الاقتصادية الكبيرة والتغيرات في بيئة الاعمال وانفتاح الاسواق المالية ادى المى زيادة الاهتحام بالاستثارات المالية لغرض انعاش الاقتصاد الوطني، ومع ظهور الاعمال الدولية والشركات متعددة الجنسيات اصبحت العمليات والاحداث التي ينبغي على المحاسبة قيامها اكثر اتساعا وتعقيدا مما يتطلب تحسين المعايير المحاسبية المحلية بها يتلاءم مع المتطلبات الدولية، بناءً على هذه الحقيقة تبلورة فكرة هذه البحث الذي يتناول موضوع المحاسبة عن الاستثمار ات المالية في ضوء معايير الابلاغ المالي الدولية والمحلية. وبها ان الاستثمارات المالية تشمل جميع الاصول المالية والالتزامات المالية وحقوق المساهمين وبهذا يجب على المنشاة تصنيف هذه الاستثلارات وقياسها والافصاح عنها في التقارير المالية، حيث تم تشريع معيار الابلاغ الملالي الدولي رقم (9) "الادوات المالية" والذي يتضمن المعالجات المحاسبية لجميع انواع الادوات المالية وبشكل منفصل. اما في العراق تم اتباع النظام المحاسبي الموحد والذي يتضمن جميع العمليات والاحداث المالية وبشكل شامل وبالمقابل تم تشريع العديد من القو اعد المحاسبية كل قاعدة تتضمن موضوع معين ومنها بصدد هذا الموضوع القاعدة المحاسبية رقم (14 ) " المحاسبة عن الاستثمارات" لغرض تحقيق اهداف البحث المتعلقة بتحديد اماكن التشابه والاختلاف بين معايير الابلاغ الملالي الدولية والمحلية بصدد الاستثارات المالية واقتراح الاسس والاجراءات اللازمة لتحسين الممارسات المحاسبية المتبعة في العراق بها يؤدي الم تقليص الفجوة و التقريب بينها. تناول المبحث الاول منهجية البحث ودراسات سابقة بينما خصص المبحث الثاني للمحاسبة عن الاستثارات المالية في ظل النظام المحاسبي الموحد والقو اعد المحاسبية العراقية. تناول المبحث الثالث الاستثمارات المالية في ظل معايير الابلاغ المالي الدولية بينها ركز المبحث الرابع على تحديد اوجه التشابه والاختلاف بين الاستثارات المالية في ضوء معايي الابلاغ الملالي الدولية والمحلية، واخيرا تناول المبحث الخامس اهم الاستنتاجات والتو صيات. 


\section{المبـحث الأول: منهجية البحث ودراسـات سـابقة \\ أو لاً: منهـهية البـحث

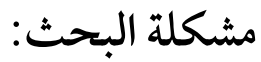

ان الاستثمارات المالية تعتبر مصدرا مهاً للدولة في زيادة ايراداتها ونمو اقتصادها وتطورها وان عدم الاهتحام بها يؤدي الى تدهور الاقتصاد وحرمانه من التمويل، ولكون العراق من الدول التي تسعى الى استخدام محاسبة تتلاءم الى حد كبير مع معايير الابلاغ الملالي الدولية من خلال حرصها على تطبيق النظام المحاسبي الموحد الذي جاء متوافقا مع معايير الابلاغ الملالي الدولية في جوانب معينة ومختلفا في جوانب اخرى، وان دراسة الاستثمارات المالية في ضوء كل من معايير الابلاغ الملالي الدولية واللوائح المحاسبية العراقية لغرض معرفة هل توجد فجوة بين معايير الابلاغ الملالي الدولية والمحلية بصدد موضوع البحث وامكانية تحديد أوجه التشابه والاختلاف بينها في ما يتعلق بالمحاسبة عن الاستثمارات المالية.

$$
\text { أهداف البحث: }
$$

1 - التعرف على الاستثمارات المالية في ضوء كل من معايير الابلاغ المالي الدولية والمحلية وبيان المعالجات

$$
\text { المحاسبية الناشئة عن انواع محددة من العمليات والاحداث الاقتصادية. }
$$

2- اجر اء دراسة تحليلية مقارنة لمعرفة وتحديد اوجه التشابه والاختلاف بين معايي الابلاغ المالي الدولية والمحلية في المحاسبة عن الاستثمارات المالية واقتراح الاسس والاجراءات اللازمة لزيادة الانسجام وفقاً للمتطلبات الدولية والمحلية.

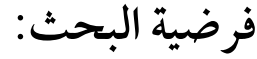

يفترض البحث "وجود فجوة بين واقع النظام المحاسبي الموحد والقاعدة المحاسبية رقم (14) وبين معايير الابلاغ الملالي الدولية في ما يتعلق بالمحاسبة عن الاستثارات المالية". 
تكمن اهمية البحث في تحسين النظام المحاسبي الموحد و القو اعد المحاسبية المحلية التي من خلالهما تتم المحاسبة عن الاستثمارات المالية واظهارها في الكشوفات المالية وبالتالي ايصال المعلومات بالشكل الصحيح ويما ينعكس على اتخاذ القرارات ويساعد في تعزيز قرارات الاستثمار وجذب المستثمرين للعمل في البيئة العراقية وانعاش الاقتصاد الوطني. منهج البحث:

استخدم الباحثان الجانب النظري والاسلوب التحليلي المقارن من خلال الاعتهاد على المصادر والمراجع فضلا عن المعايير والقواعد المحاسبية الصادرة عن بجلس المعايير المحاسبية والرقابية العراقي ومعايير الابلاغ الملالي المتعلقة بموضوع البحث، حيث تم عرض وتوضيح اهم الاستثمارات والادوات المالية في ضوء كل من معايير الابلاغ المالي الدولية والمحلية وذلك باستخدام ادوات ايضاحية كالأشكال والجداول. طرق جمع البيانات والمعلومات: 1 - الاستعانة بالمصادر من الكتب والوثائق و الدوريات المنشورة العربية والاجنبية. 2 - الاطلاع على البحوث والرسائل والاطاريح ذات الصلة بالموضوع. 3 - القو انين و التعليمات الصادرة ومعايير الابلاغ المالي الدولية والمحلية.

\section{ثانيا : دراسـات سـابقة}

يتناول هذا المحور بجموعة من الدراسات السابقة التي تناولت موضوع الاستثمارات المالية وذلك لغرض عرضها والاستفادة منها لإعداد لهذا البحث. دراسة ابو بكر واخرون، 2019

الموسومة ( معايير تقييم قرارات الاستثمار ات في اسواق الاوراق المالية)

تعد السوق المالية سوق يلتقي فيها البائعون والمشترون لتداول الادوات المالية، الاستثمار هو تخصيص الاموال في مجالات مختلفة بشكل يؤدي المى تعظيم المردودية الاقتصادية والمالية والغرض من هذه الدراسة هو توظيف 
المتاح من المعلومات حول اتخاذ قرارات الاستثلار في اسواق الاوراق المالية، حيث تكمن مشكلة الدراسة في قصور معدلات الادخار بمختلف انو اعه عن معدلات الاستثمار فتمويل التنمية يكمن في البحث عن مصادر الادخار والبحث عن الوسائل الكفيلة بتعبئة هذه المدخرات لأغراض التنمية ولا يتم ذلك الاعن طريق توفر هيكل متكامل من المؤسسات المالية والتنظيات القادرة على التحريض والتوجيه الجميد لهذه المدخرات لأغراض الاستثمار المتنج، حيث تم طرح الاشكالية التالية: ما هي معايير تقييم القرارات الاستثلارية في الاسو اق المالية؟ حيث تناولت هذه الدراسة مفهوم الاستثار المالي وسوق الاوراق المالية وكذلك تضمن تقييم قرارات الاستثمار في اسواق الاوراق المالية واهم المؤشرات المستعملة في عملية تقييم الاستثمارات المالية، حيث توصلت هذه الدراسة الى ان السوق المالية تنشط بمعية الادوات المالية المثداولة فيها، الطريقة التي تتم بها عملية التداول، البيئة التي تتم فيها العملية الاستثمرية او ما يعرف بالاستثلار المالي كما ان التنويع في الاستثمارات يؤدي الى تقليل المخاطر حيث انه كلما اتسعت مساحة التنوع ادى ذلك الى تقليص حجم المخاطر.

\section{دراسة نصار واخرون، \\ الموسومة ( خخاطر الاستثمار في الاوراق المالية )}

السوق المالية اداة تفاعل ومكان التقاء قوى الاستثلار المختلفة واداة تمويلية بالنسبة للدول المتطورة لتمويل اقتصادها، حيث هدف البحث الى تحديد مفهوم الاستثلار الملالي وبجالاته وعرض ادواته بالإضافة الى التعرف على نخاطر الاستثلار في الاوراق المالية واساليبها، تم استخدام المنهج الوصفي التحليلي وقد تم التوصل على ان للسوق المالية دور فعال في تعبئة الادخار وتوجيه نحو الاستثمار وذلك من خلال جلب المستثمرين وبث حركة نشيطة في السوق. دراسة معروف والعتابي، 2019 الموسومة ( اثر تداول اسهم غير العراقيين في الاستثمارات المالية) 
تعد المؤسسات المالية جزءا من النظام الملالي الذي يخدم المجتمع حيث يتكون النظام الملالي من شبكة من المؤسسات المالية والاسواق ورجال الاعحال والافراد والحكومة التي تشارك في هذا النظام وتنظم عملياته، حيث يهدف البحث الى مدى تأثير حجم تداول غير العراقيين للاسهم في سوق العراق للأوراق المالية على حجم الاستثمار الملالي الكلي في السوق ويفرض البحث وجود علاقة ذات دلالة احصائية بين متغير حجم تداول غير العراقيين وبين حجم التداول الكلي للسوق واتخذ البحث سوق العراق للأوراق المالية عينة البحث كونه المكان الوحيد لتداول الاسهم في العراق، حيث استنتج البحث وجود تأثير ايجابي لحجم التداول لغير العراقيين في حجم الاستثمار الملالي ومن توصيات البحث تشجيع الاستثمار الاجنبي من اجل معالجة الانخفاض الحاد في حجم تداول المستثمرين الاجانب في العراق وذلك لما له من اهمية على مستوى الاقتصاد العراق ككل والسوق بصورة خاصة.

\section{دراسة محمد، 2008}

الموسومة ( تحليل البيئة الاستثمارية للاستثمار الملالي الاجنبي في العراق) توفر الاستثمارات الاجنبية والمالية المال اللازم للاستثمار الوطني اذ يمكن ان تعد مصدرا للتمويل وذلك من خلال اقتنائها للاسهم المطروحة في السوق المالية الوطنية ولعدم معرفة هذه الاستثمارات بالبيئة الاستثمارية الوطنية فأنها تتجنب الاستثمار في بيئة غير معروفة، حيث هدف البحث الى تحليل البيئة الاستثمارية في العراق من الناحية الامنية والاقتصادية والمالية وتحديد الفرص الاستثمارية المتاحة، ان تحليل البيئة الاستثمارية وتحديد الفرص الاستثمارية المتاحة والقطاعات المناسبة يعد عامل جذب الاستثمارات المالية، حيث استنتج البحث على افتقار البيئة الاستثمارية في العراق الى الامن والاستقرار السياسي والاقتصادي حيث لم تتضح العملية السياسية والاقتصادية ووجود فرص استثلارية في الاقتصاد العراقي والقطاع الزراعي والمو اصلات والخدمات، يوصي الباحثان بتشجيع الاستثمار بها يضمن حقوق البلد وسيادته وتوفي عوامل الجذب القانونية. 


\section{دراسة عنيزة و النصر اوي، 2016}

الموسومة (تكييف متطلبات البيئة الوطنية لتطوير القواعد المحاسبية وتحقيق التوافق مع المعايير المحاسبية

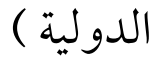

هدف البحث المى تسليط الضوء على مفهوم المعايير المحاسبية الدولية واهميتها وكذلك التعرف على القواعد المحاسبية العراقية والجهات القائمة على اعدادها ومن ثم التوصل الى حلول مقترحة عن مدى امكانية توافق القواعد المحاسبية العراقية مع معايير المحاسبة الدولية، حيث اعتمد الباحثان على الاسلوب الاستنتاجي في صياغة المشكلة وفرضيتها والاسلوب التحليلي الوصفي لتغطية البحث، وقد تم التوصل الم ان تخلف القواعد المحاسبية وعدم تفاعلها مع متطلبات المرحلة ناتج من واقع لا يضع اهتمام للمحاسبة وقو اعدها وبذلك تعاني من التقدم وعدم التحديث، بالإضافة الى ضرورة اعتماد المعايير الدولية في صناعة وتطوير القواعد المحاسبية العراقية بو صفها اداة لاستقطاب المستثمرين. ان اهم ما يميز البحث الحالي عن الدراسات السابقة: 1- تناول البحث معايير الابلاغ الملالي الدولية والنظام المحاسبي الموحد والقواعد المحاسبية العراقية الخاصة بموضوع المحاسبة عن الاستثمارات المالية. 2- اجراء دراسة تحليلية مقارنة لغرض ايجاد اماكن التشابه والاختلاف بين معايير الابلاغ الملالي الدولية والمحلية ذات العلاقة بموضوع الاستثمارات المالية واقتراح الاساليب والاجراءات اللازمة لزيادة الانسجام بينها. 


\section{المبـحث الثاني: المحاسببة عن الاستثمارات المالية فِّ ظل المعايير والقواعد المحاسبيية}

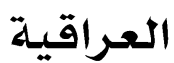

\section{أو لا : القاعدة المحاسبية رقم (14) محاسبة الاستثمهارات}

اقر بجلس المعايير المحاسبية والرقابية في جمهورية العراق بجلسته المنعقدة بتاريخ 7/5/ 2001 القاعدة المحاسبية التالية بشأن محاسبة الاستثمارات.

\section{التعريف بالاستثمـارات المالية وانواعها}

الاستثارات هي احد الاصول التي تحتفظ المنشاة بها لغرض زيادة مواردها او تنمية الثروات، من خلال ما تحصل عليه من توزيعات على شكل فوائد او عوائد من حقوق الامتياز وتوزيعات الارباح او العائد من الايجارات، لغرض ارتفاع قيمثه الرأسمالية او لغرض الحصول على منافع مماثلة لتلك التي تحصل عليها من خلال العلاقات التجارية. (www.bsairaq.net). حيث شملت الاستثارات قصيرة الاجل (المتداولة) التي تتصف بقابليتها العالية للتسويق مما تكسبها درجة عالية من السيولة، ان تكون نية الاحتفاظ بها لا تزيد عن سنة وتشمل هذه الاستثمارات (محلية ودولية) كلاً من الودائع الزمنية والاوراق المالية (الاسهم والسندات) ، والاستثمارات طويلة الاجل اي استثمار عدا الاستثمار المتداول الذي تزيد مدة الاحتفاظ به عن سنة، والاستثمار في العقارات يشمل الاستثمارات في الاراضي والمباني غير المشغولة وغير المستخدمة بصورة رئيسية في عمليات المنشاة المستثمرة. (www.bsairaq.net). يجب على المنشاة التمييز بين الاستثمارات قصيرة الاجل والاستثمارات طويلة الاجل على ان تعرض الاولى ضمن الاصول المتداولة والثانية ضمن الاصول طويلة الاجل. (نجم وناصر، 2011: 18 -19 )

\section{القيملة المدرجة للاستثمهارات}

الاستثمارات قصيرة الاجل (المتداولة)

$$
\text { يتم معالجة الاستثمارات القصيرة الاجل وفق الاتي (نجم وناصر، 24:25 2011 ) }
$$


يجب ان تدرج الاستثمارات قصيرة الاجل كموجودات متداولة في الميزانية العامة بسعر الكلفة او السوق ايهها

$$
\text { اقل في تاريخ الميزانية. }
$$

1- في حال كون الكلفة اعلى من القيمة السوقية لمحفظة الاستثار المثداولة لاسهم الملكية فان الفرق يتم اتخاذه كأساس لتكوين خخصص هبوط في القيمة السوقية ،تسجيل الخسائر غير المتحققة في تاريخ اعداد

$$
\text { الميزانية في حين لا توجد المكاسب غير المحققة كايراد. }
$$

2- ان تقلبات اسعار سوق الاسهم التي في صالح المنشاة تتم المحاسبة عليها نتيجة لاختيار القيمة السوقية

$$
\text { في تاريخ اعداد الميزانية العامة. }
$$

3- يتم غلق حساب الخسائر غير المحققة في حساب الارباح والخسائر اما خصص هبوط القيمة السوقية

\begin{tabular}{|c|c|c|c|}
\hline ارباح (خسائر) غير معقة & سوينار & كلفة & التفاصيل \\
\hline (500) & 1500 & 2000 & شاستمارات في اسهم \\
\hline 1000 & 8000 & 7000 & 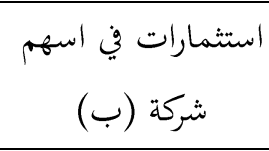 \\
\hline (4000) & 2500 & 6500 & 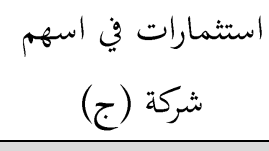 \\
\hline$(3500)$ & 12000 & 15500 & الجموع \\
\hline$(3500)$ & & & المخصص المطلوب \\
\hline
\end{tabular}
يظهر مطروحاً من الاستثمارات المثداولة في الميزانية العامة.

مثال: تتضمن محفظة الاستثمارات المتداولة ما يلي: (www.bsairaq.net).

ويتبين المثال اعلاه ان الاستثمار في اسهم الشركة ( أ ) نتجت خسائر غير محققة بمقدار (500) دينار وذلك لكون الكلفة اعلى من القيمة السوقية، بينما الاستثلارات في الشركة (ب) تضمنت ارباح غير محقة بمقدار (1000) الف دينار لكون الكلفة اقل من القيمة السوقية. (اعداد الباحثان بالاعتماد على بيانات الجدول 


\section{الاستثمارات طويلة الاجل}

يتم معالجة الاستثمارات طويلة الاجل وفق الاتي (www.bsairaq.net).

1 - ان تدرج الاستثمارات المصنفة كموجودات طويلة الاجل في الميزانية العامة بالكلفة.

2- يتم تحميل حساب الارباح والخسائر بمبلغ اي انخفاض غير مؤقت في القيمة المدرجة للاستثمارات

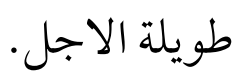

3 - عند حدوث انخفاض غير مؤقت في قيمة الاستثمار ات طويلة الاجل يتم في هذه الحالة تحفيض قيمتها المدرجة لغرض الاعتراف بالخسارة الناتجة عن الانخفاض.

\section{الافصاح عن الاستثمارات المالية}

$$
\text { يتم الافصاح عن الاستثمارات المالية وفق الاتي: (نجم وناصر، }
$$

1 - يستلزم الافصاح عن السياسة المحاسبية لغرض تحديد القيمة المدرجة للاستثمارات.

2- يجب الافصاح عن القيمة الدفترية للاستثارات العقارات (الكلفة - خصص الاندثار المتراكم) ضمن

$$
\text { الموجودات الثابتة. }
$$

$$
\begin{aligned}
& \text { 3- يجب الافصاح في حساب الارباح والخسائر عن ما يلي: } \\
& \text { أ. ايرادات الاستثمارات المتأتية من : }
\end{aligned}
$$

الفوائد ،العوائد وتوزيعات الارباح ، الايجارات سواء من الاستثمارات المتداولة او طويلة

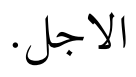

الارباح والخسائر الناتجة من بيع او اعادة تصنيف الاستثمارات المتداولة.

الهبوط في القيمة السوقية لغرض اظهار الاستثمارات المتداولة في الكلفة او السوق ايها اقل .

$$
\text { ب. الهبوط غير المؤقت في قيمة الاستثمارات طويلة الاجل . }
$$

ت. الارباح والخسائر الناتجة من بيع او اعادة تصنيف الاستثمرات طويلة الاجل . 4 - الافصاح والتحليل في تقرير الادارة عن معلومات: (www.bsairaq.net). 


$$
\text { أ. القيمة السوقية للاستثمارات القابلة للتداول . }
$$

ب. القيمة العادلة للاستثمارات العقار ات عند اعتبارها كاستثمارات طويلة الأجل . ت. القيود الهامة المفروضة على الاستثارات لغرض تحويل عو ائدها والمتحصلات من بيعها . ث. تحليل محافظ الاستثمار ات بالنسبة للمنشآت التي يكون عملها الرئيسي التعامل بالاستثمارات . ج. تحليل محافظ الاستثمار الطويل الاجل حسب اصنافها. ح. تفاصيل اي استثمار يمثل بمفردة نسبة هامة من بجموع موجودات المنشاة. خ. اية امتيازات تتمتع بها المنشاة بسبب الاستثمارات كعضوية بجالس الادارة.

\section{ثانيًا : المحاسبة عن الاستثمارات المالية يِّ ظل النظام المحاسبي الموحد}

\section{التعريف بالاستثـمارات المالية وانواعها}

الاستثمارات المالية تشمل حركة الاستثمارات المختلفة التي تقوم بها الوحدة الاقتصادية بهدف استغلال فائض السيولة النقدية لديها عن طريق الودائع الثابتة لدى المصارف وشراء الاسهم والسندات والعقارات التي تشتري بهدف تحقيق العوائد. (النظام المحاسبي الموحد، 2011: 69 6). والجمدول ادناه يبين انواع الاستثمارات المالية وتصنيفها حسب كل قطاع. 


\begin{tabular}{|c|c|c|c|}
\hline \multirow[t]{2}{*}{ 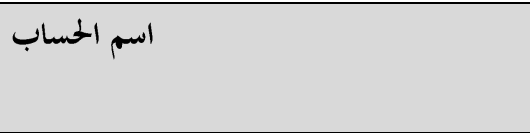 } & \multicolumn{3}{|c|}{ رقم الدليل على المستوى } \\
\hline & | الرباعي & الثلاثي & الثنائي \\
\hline | استثمارات مالية & & & 15 \\
\hline استثمارات مالية طويلة الاجل & & 151 & \\
\hline استثمارات طويلة الاجل في القطاع الاشتراكي & 1511 & & \\
\hline استثمارات طويلة الاجل في القطاع التعاوتي & 1512 & & \\
\hline استثمارات طويلة الاجل في القطاع المختلط & 1513 & & \\
\hline استثمارات طويلة الاجل في القطاع الخاص & 1514 & & \\
\hline استثمارات طويلة الاجل في القطاع الخارجي & 1515 & & \\
\hline استثمارات مالية قصيرة الاجل & & 152 & \\
\hline استثمارات قصيرة في القطاع الاشتراكي & 1521 & & \\
\hline استثمارات قصيرة في القطاع التعاوني & 1522 & & \\
\hline |ستثمارات قصيرة في القطاع المختلط & 1523 & & \\
\hline استثمارات قصيرة في القطاع الخاص & 1524 & & \\
\hline استثمارات قصيرة في القطاع الخارجي & 1525 & & \\
\hline
\end{tabular}

المصدر: (من اعداد الباحثين بالاعتماد على النظام المحاسبي الموحد)

تحلل الاستثمارات المالية الى قسمين رئيسين: (النظام المحاسبي الموحد ، 2011 : 69 ) 1 - استثمارات طويلة الاجل

يبين هذا الحساب الاستثمار ات في الاسهم والسندات والعقارات التي تنشى بهدف تحقيق العوائد والتي تمتد فترة المساهمة لمدة طويلة.

$$
2 \text { - استثمارات قصيرة الاجل }
$$

تمثل ما تقوم به الوحدة الاقتصادية من استثمار للسيولة النقدية الفائضة لديها عن طريق الودائع النقدية الثابتة لدى المصارف وشر اء الاسهم و السندات والعقارات. 


\section{المعالجات المحاسبيية للاستثمارات المالية}

تعد المعالجات المحاسبية جزءا مكملا لشروحات الدليل المحاسبي اذ تمثل الجانب التطبيقي له ويعتمد النظام المحاسبي الموحد على اساس الاستحقاق في اثبات قيود العمليات المالية التي تحدث في الوحدة الاقتصادية وخاصة في نهاية السنة المالية. (احمد ومحمود، 2009 : 337 )

وفيما يأتي المعالجات المحاسبية للاستثمارات المالية في الوحدات الاقتصادية: (النظام المحاسبي الموحد، 2011:

1 - استثمارات قصيرة الاجل: تدرج كموجودات متداولة في الميزانية العامة بسعر الكلفة او السوق ايهها اقل في تاريخ الميزانية ولكون الكلفة اقل من القيمة السوقية للاسهم المتداولة فيعترف بخسائر هبوط القيمة السوقية مقابل خصص هبوط القيمة السوقية للاستثمارات المتداولة وتعالج هذه الخسائر في حساب خسائر هبوط الاستثارات المالية ويعرض المخصص مطروحا من الاستثمارات المتداولة في الميزانية. 2 - استثمارات طويلة الاجل: فيعترف بخسائر هبوط القيمة غير المؤقت ويعالج في حساب خسائر هبوط اسعار الاستثمار ات المالية ويجري تخفيض قيمة الاستثلارات طويلة الاجل مباشرة.

\section{المبـحث الثالث: الاستثمهارات المالية ِِفِ ظل معايير الابلاغ المالي الدولية أولا : التعريف بالأدوات المالية وانواعها} عرفت الادوات المالية بتعاريف متعددة منها: الادوات المالية: هي اي عقد ينشأ عن الموجودات المالية للمنشأة والمطلوبات المالية على المنشاة او حقوق مساهمين لمنشات اخرى. (خليل، الادوات المالية: انها حق ملكية اي عقد يدل على حصة في صافي الاصول ويقصد بصافي الاصول الحصة المتبقية في اصول منشاة بعد خصم كل التزاماتها ويلاحظ ان الالتزام بإصدار اداة حقوق الملكية ليس التزاما 
ماليا لأنه ينتج عنه زيادة في حقوق الملكية ولا يمكن ان يترتب عليه خسارة المنشاة. (المجمع العربي للمحاسبين القانونيين، 2007).

الاستثمار المالي: بانه الاستثمار المتعلق بالأسهم والسندات واذونات الخزينة والادوات التجارية والقبولات

$$
\text { المصرفية والودائع القابلة للتداول والخيارات. (محمد، } 2019 \text { 2: 390). }
$$

ويرى الباحثان ان الاستثمارات المالية تعني الادوات المالية القانونية التي تمنح حاملها منافع وعوائد وامتيازات في فترة زمنية وبشروط معينة.

هناك عدة انواع للاستثمارات في الو اقع العملي يمكن عرضها بالشكل التالي:- (من اعداد الباحثان بالاعتماد

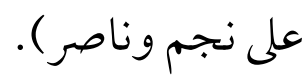

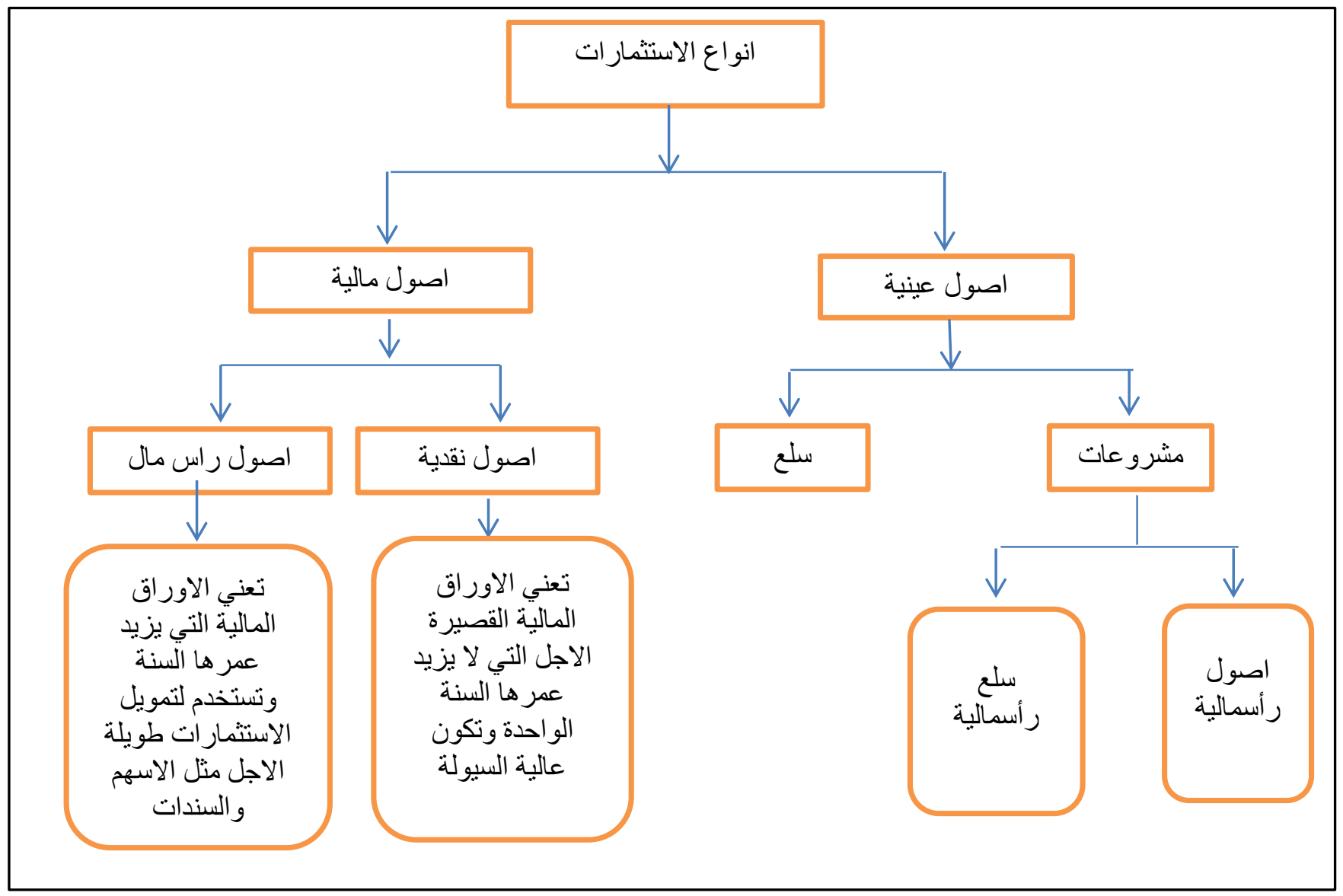

$$
\text { الشكل رقم (1 ) تصنيف الاستثمارات }
$$


يلاحظ من الشكل اعلاه ان الاستثمارات تصنف الى اصول عينية واصول مالية فالأصول العينية هي كل اصل له قيمة اقتصادية ويترتب عليه منفعة اضافية سلعية او خدمية فمثلا العقار اصل عيني، بينما الاصول المالية لا يترتب لحاملها حق حيازة اصل حقيقي وانما توفر لمالكها حق المطالبة بالحصول على عوائد كالفو ائد والارباح. (من اعداد الباحثان بالاعتماد على نجم وناصر) ).

يمكن تصنيف الادوات المالية التقليدية من حيث الاستحقاق الم: (عبدالصمد، 20 2: 66 ) 1 - ادوات مالية قصيرة الاجل: يتم انجازها وجني عو ائدها في مدة تمتاز بالقصر نسبيا حيث تتراوح المدة بين سنة الى سنتين وتعتبر الاكثر انتشارا في الاستثمارات المالية مثل الودائع الزمنية والتسهيلات الائتحانية. 2 - ادوات مالية متوسطة الاجل: يتم انجازها في فترة لا تقل عن سنتين ولا تزيد عن سبع سنوات وتشمل

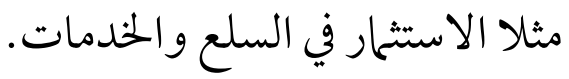
3 - ادوات مالية طويلة الاجل: يتم انجازها في مدة تفوق سبع سنوات وتشمل الاصول والمشروعات الاقتصادية التي تنشأ لأجل تشغيلها والاستفادة منها لمدة طويلة نسبيا مثل المشاريع العقارية. والجدول ادناه يوضح الاوراق المالية المتداولة في سوق النقد وسوق راس المال: جدول رقم (2) الاوراق المالية المتداولة

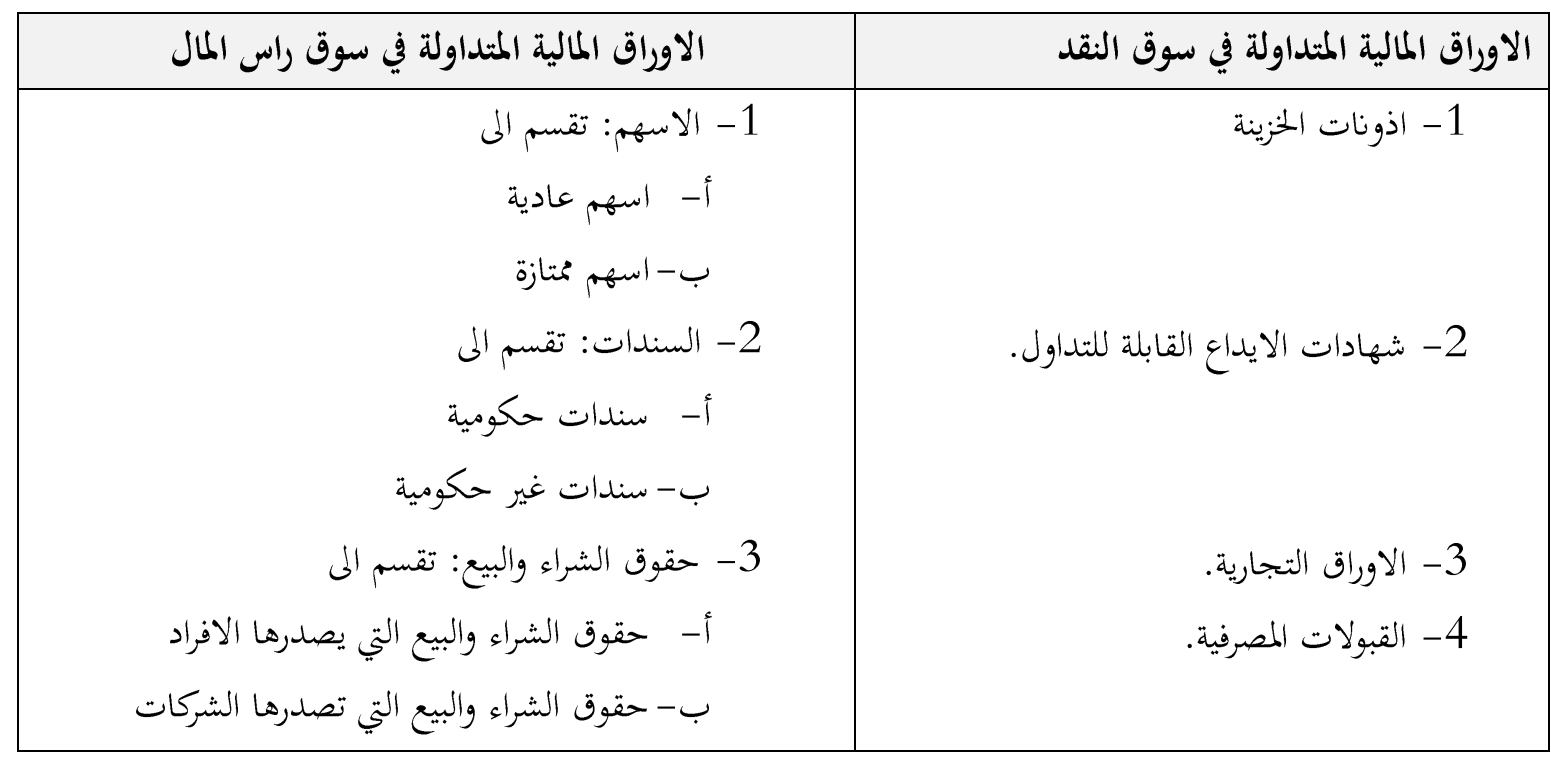

$$
\text { المصدر ( من اعداد الباحثان بالاعتماد على عبدالصمد). }
$$


من خلال الجمدول اعلاه فان الادوات المالية تقسم الم: -

1 - الاسهم: تمثل حصة في ملكية المنشاة ويتمتع المساهم بحقوق الملكية ومن بينها الحق في حصة من الارباح التي تحققها المنشاة والحق في مراقبة الادارة والتصويت وتنقسم الاسهم بحسب طبيعة الحصة

$$
\text { التي يقدمها كل شريك الى اسهم عادية واسهم ممتازة.(توفيق، } 2088 \text { 2: } 17 \text { - 20 ). }
$$

2 - السندات: عبارة عن صكوك مديونية على الجهة التي اصدرتها طويلة الاجل قابلة للتداول يحصل حاملها على سعر فائدة خلال مدة استحقاقها على ان يسترد قيمة السندات عند ميعاد استحقاقها. (ابو بكر واخرون، 2019: 14 ) ) وتقسم السندات المى نوعين هما سندات حكومية وسندات غير حكومية. 3 - اوراق الديون: هي ادوات تمثل علاقة دائن معين بمنشاة وتتضمن اوراق الديون الحكومية وسندات الشركات والديون القابلة للتحويل والاوراق التجارية وادوات الديون المضمونة. ولا تعتبر حسابات المدينين التجارية وحسابات الاقراض للغير ضمن اوراق الديون لأنها لا تستوفي تعريف الورقة المالية ويمكن تجميع الاستثمارات في اوراق الديون الى ثلاث بجموعات منفصلة لأغراض منفصلة والتقرير المالي (2015: 952،weygandt‘kieso).

\section{ثانيا : المعالجات المحاسبية للاستثمهارات المالية وفق معايير الابلاغ المالي الدولية} يتم الاعتراف بجميع الاصول المالية والالتزامات المالية بالرغم ان المنشاة لا تكون قد دفعت او قبضت شيئا عند الدخول في الادوات المالية عندها تصبح المنشاة طرفا في اشتراطات تعاقدية لأداة ما من اجل شراء او بيع اصول مالية حيث تحدد اتفاقية السوق مدة ثابتة بين تواريخ التعامل والتسوية ـ (وسام، 110145 2 14 ). في ظل القواعد التي اصدرها بجلس معايير المحاسبة الدولية فان الاستثمارات في الاوراق المالية الخاصة بالملكية والممسوكة لحين الاستحقاق تسجل بالكلفة المطفأة وجميع الانواع الاخرى تسجل بالقيمة العادلة لها (اذا كان القيمة العادلة غير موثوق بها يجب ان تسجل بالكلفة). (الشمري والمعيني، 140 2: 237 ). 


\section{وللأغراض المحاسبية ينبغي ان نميز بين ثلاثة انواع من الاستثارات المالية: 1 - الاستثلارات الممسو كة حتى تاريخ الاستحقاق}

تصنف اوراق المديونية فقط ضمن هذا التصنيف لان الاوراق المالية الخاصة بالملكية ليست لها تواريخ استحقاق محددة، فضلا عن ذلك فان اوراق المديونية تصنف ضمن هذا النوع من الاستثارات اذا كانت وحدة الابلاغ (الشركة) لديها النية الكاملة والقدرة على الاحتفاظ بهذه الاوراق حتى تاريخ الاستحقاق. وعلى عكس الاسهم فان السندات او اوراق المديونية الاخرى لديها تواريخ محددة للاستحقاق، وفي تاريخ استحقاقها فان القيمة الاسمية يجب ان تدفع للمستثمر. وفي مدد فاصلة هناك فوائد تمثل نسبة من القيمة الاسمية تدفع الى المستثمر ايضا. فاذا كانت معدلات الفائدة في السوق اعلى من معدلات الفائدة الثابتة للأوراق المالية المشتراه فان القيمة السوقية للاستثمارات وعلى عكس ذلك فاذا انخفضت معدلات الفائدة السوقية عن المعدل الثابت للأوراق المالية فان مدفوعات الفائدة عن الاوراق المالية تكون اعلى من الفائدة السوقية، وبذلك ترتفع الاسعار السوقية لتلك الاستثمارات.(الشمري والمعيني، 225:2010) حيث يتم الافصاح عن الاستثمارات اعلاه بالكلفة المسجلة. 2- الاستثمارات المالية المتاحة للبيع يتم التقرير عن الاستثمارات التي تصنف ضمن هذا المجموعة على اساس قيمتها العادلة. كما تسجل المكاسب والخسائر غير المتحققة المرتبطة بالتغيرات في القيمة العادلة لهذه الاسثثارات في حساب مكاسب او خسائر حيازة غير متحققة ويقرر عن هذه الخسائر كجزء مستقل ضمن حقوق المساهمين لحين تحقق هذه المكاسب او الخسائر و وعلى ذلك فان التغيرات في القيمة العادلة لا يقرر عنها كجزء من صافي الدخل لحين بيع هذه

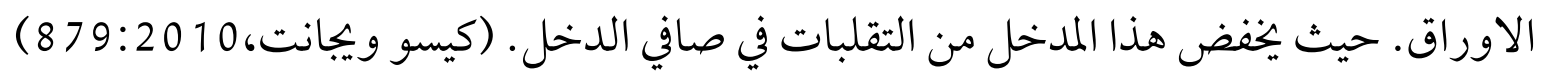
3- الاستثارات الممسو كة لأغراض المتاجرة يجب على المنشاة تحديد ما اذا كان هدف نموذج اعحال المنشاة لإدارة اصل مالي يستوفي الشروط او اذا كان الاصل المالي مؤهلاً للاختيار وذلك الغرض يجب على المنشاة تحديد ما اذا كانت الاصول المالية تستوفي تعريف 
عتفظ بها للمتاجرة اذا كانت المنشاة قامت بشراء الاصول في تاريخ سابق، تعكس المتاجرة عموما الشراء والبيع النشط والمتكرر وعموما تستخدم الادوات المالية المحتفظ بها للمتاجرة بهدف توليد ربح من التذبذب قصير الاجل في السعر او هامش المتعامل. (المعايير الدولية لأعداد التقارير المالية، 2016: 94- 95 95 ). عند بيع استثمار معين من المحفظة فان الفرق بين سعر البيع والكلفة يتم الاعتراف به كمكاسب او خسائر بيع

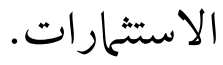

\section{ثالثا : عرض الاستثمارات بالقوائم المالية

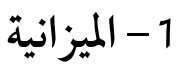

تلتزم الشركات بعرض القيم الفردية للأنواع الثلاثة من الاستثلارات سو اء في الميزانية او في الملاحظات الواردة

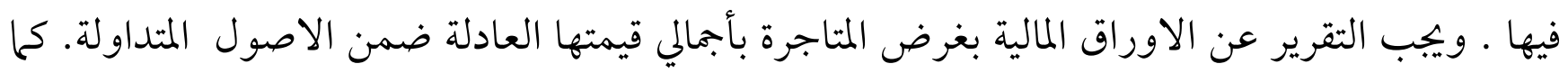
تصنف الاوراق المالية المقتناة حتى الاستحقاق والاوراق المتاحة للبيع كأصول متداولة او غير متداولة بناءا على الظروف المحيطة. ( كيسو وويجانت، 2010 : 900 )

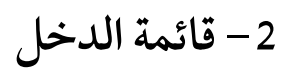

تفصح الشركات عن ايراد الفائدة ومقسوم الارباح المستلم ومكاسب او خسائر الحيازة الغير المتحققة وتسوية القيمة العادلة بشكل منفصل في كشف الدخل تحت تسمية ايرادات الفوائد والدخل. ( الشمري والمعيني، .$(237: 2014$ 


\section{المبـحث الرابع: أماكن التقارب والاختـلاف كلاستثمارات المالية فِ ظل معايير الابلاغ المالي}

لغرض اختبار فرضية البحث وبيان نتائجهُ تم عمل جدول يتضمن اسس المقارنة بين معايير الابلاغ الملالي الدولية وما ورد في النظام المحاسبي الموحد والقواعد المحاسبية العراقية في ما يتعلق بالمحاسبة عن الاستثمارات المالية لبيان اماكن التقارب والاختلاف بينها وذلك لأثبات صحة فرضية البحث من عدمها.

جدول (3) اماكن التقارب والاختلاف بين معايير الابلاغ المالي الدولية والمحلية للاستثمارات المالية

\begin{tabular}{|c|c|c|c|c|}
\hline الاستنتاج & معايير الابلاغ المالي الدولية & النظام الخحاسبي الموحد والقواعد الخحاسبية & اساس المقارنة & ت \\
\hline جوهري اختلاف & 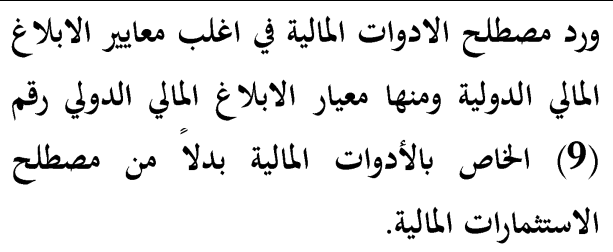 & 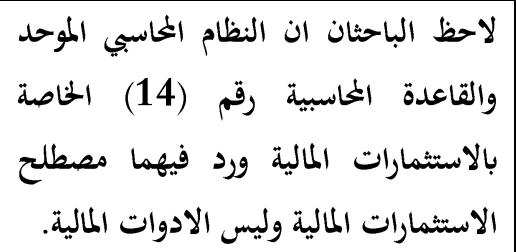 & 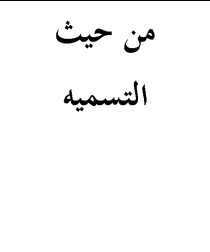 & 1 \\
\hline اختلاف & 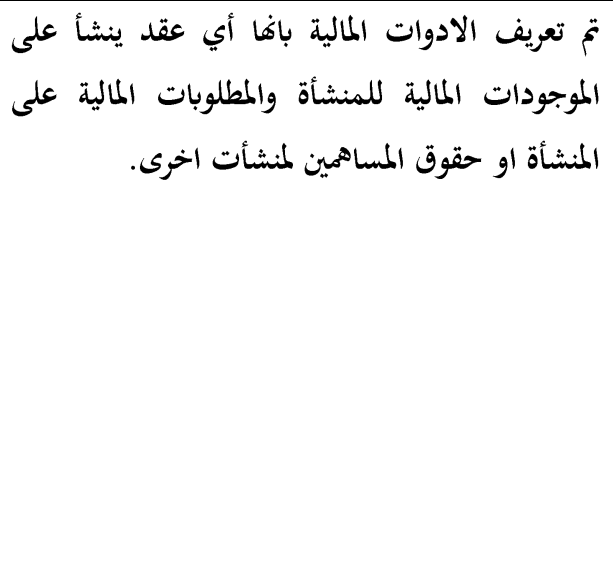 & 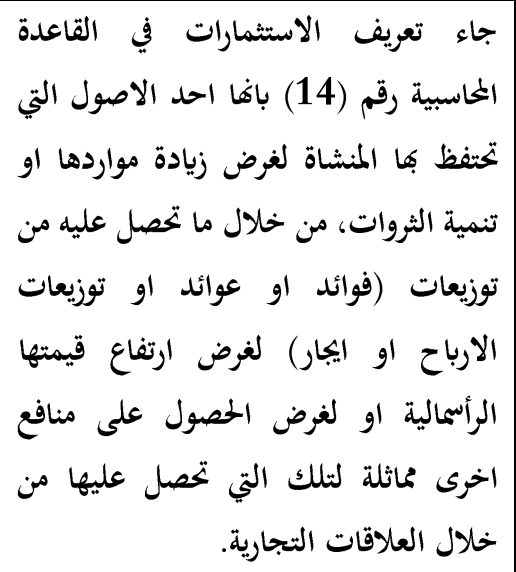 & من حيث تعريف & 2 \\
\hline جوهري & 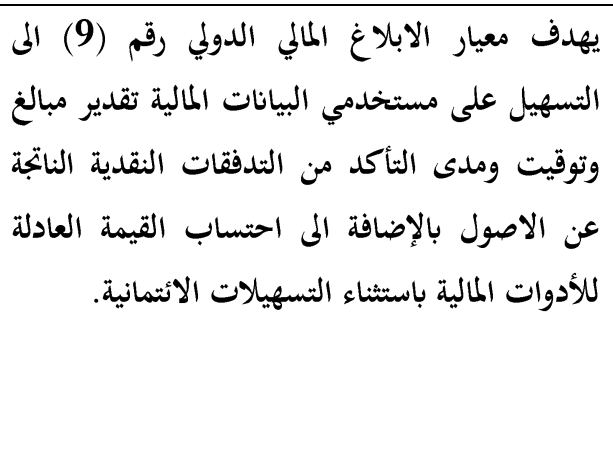 & 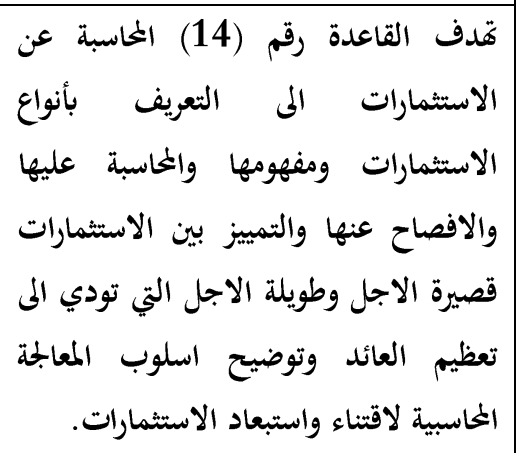 & من حيث & 3 \\
\hline اختلاف & يجب ان يطبق معيار الابلاغ المالي رقم (9) من قبل & 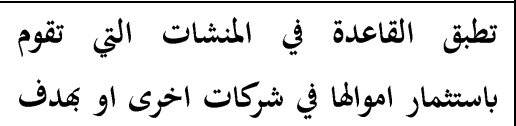 & 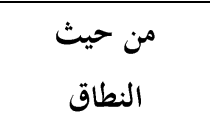 & 4 \\
\hline
\end{tabular}




\begin{tabular}{|c|c|c|c|c|}
\hline & التامين. & تحقيق الارباح والحصول على الايرادات & & \\
\hline جوهري & 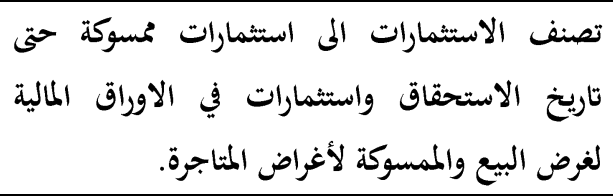 & تصنف الاستثمارات المى استثمارات قصيرة & من حيث الاستثمارات & 5 \\
\hline جوهري اختلاف & 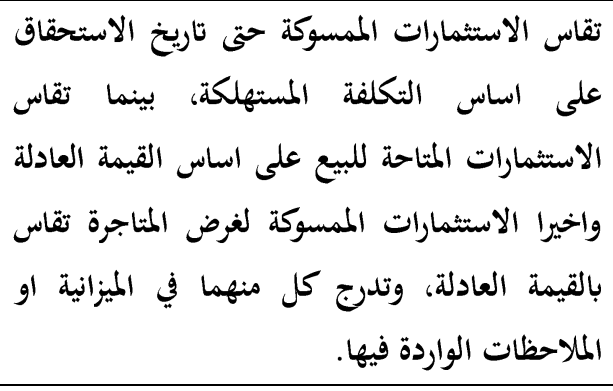 & 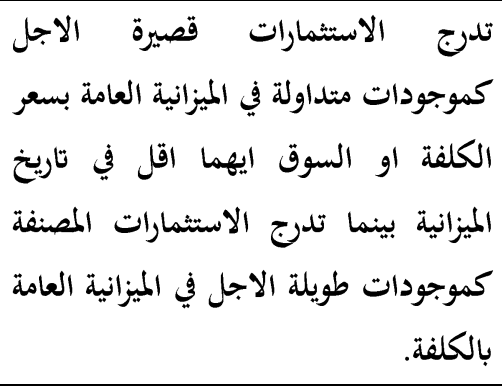 & القياس والاثبات & 6 \\
\hline اختلاف & 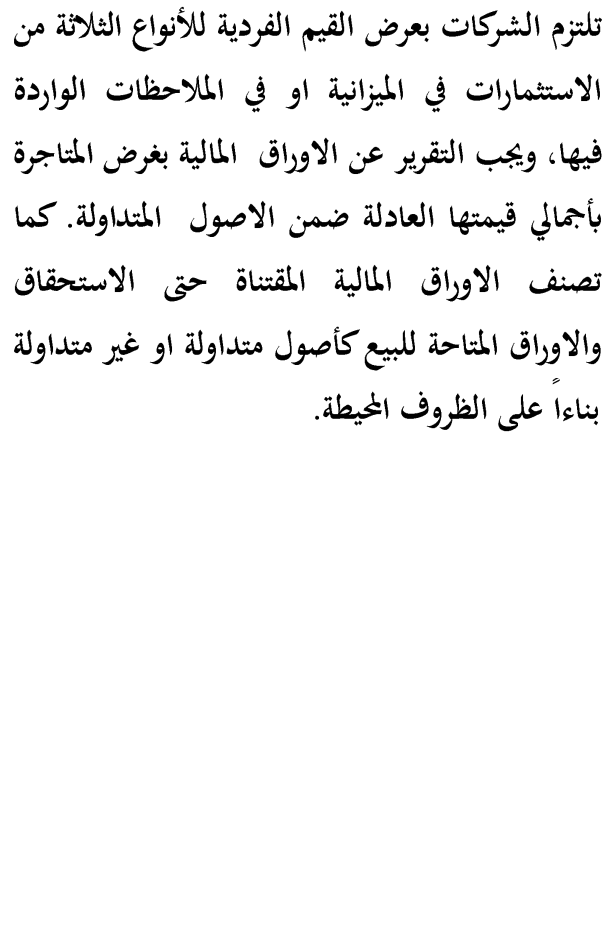 & 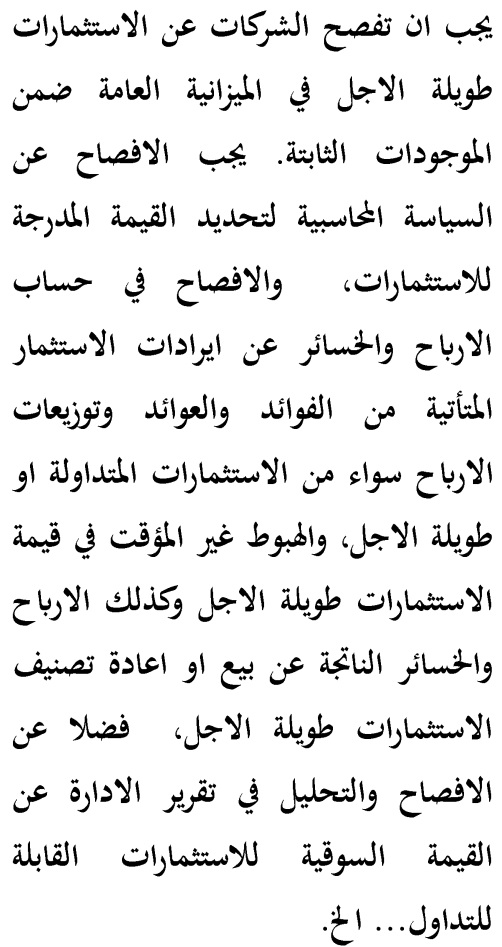 & في الافصاح عنها & 7 \\
\hline
\end{tabular}

$$
\text { (من اعداد الباحثين) }
$$

من خلال جدول المقارنة اعلاه يتبين ان هناك فروق جوهرية في جوانب معينة وحسب اسس المقارنة المستخدمة، فمثلا في ما يتعلق بتعريف الاستثارات المالية في ضوء القاعدة المحاسبية العراقية (14 ) نلاحظ انها شملت جميع الاصول وبشكل عام دون الاخذ بالالتزامات المالية وحقوق الملكية على عكس ما ورد في 
تعريفها في ضوء معايير الابلاغ المالي الدولية حيث تضمنت الاصول المالية للمنشاة والالنزامات المالية على المنشاة وحقوق المساهمين لمنشأت اخرى. اما في ما يتعلق بنطاق التطبيق فان القاعدة المحاسبية رقم (14) والخاصة بالمحاسبة عن الاستثمارات قد شملت جميع الاستثمارات على عكس ما ذكر في معايي الابلاغ الملالي والتي خصصت معيار محاسبي للأدوات المالية ومعيار اخر للأدوات المالية في الشركات الزميلة والتابعة ومعيار محاسبي اخر للعرض والافصاح عن الادوات المالية ولكل منها معالجات محاسبية تختلف عن الاخرى حسب الحالة. اما من حيث القياس والاثبات تدرج الاستثمارات قصيرة الاجل كموجودات متداولة في الميزانية العامة بسعر الكلفة او السوق ايها اقل في تاريخ الميزانية بينها تدرج الاستثمارات المصنفة كموجودات طويلة الاجل في الميز انية العامة بالكلفة حسب القاعدة المحاسبية رقم (14 )، بينما تقاس الاستثمارات الممسوكة حتى تاريخ الاستحقاق على اساس التكلفة المستهلكة، وتقاس الاستثمارات المتاحة للبيع على اساس القيمة العادلة واخيرا الاستثمارات الممسوكة لغرض المتاجرة تقاس بالقيمة العادلة، وتدرج كل منها في الميزانية او الملاحظات الواردة فيها حسب معايير الابلاغ المالي الدولية، وبهذا ثبتت صحة فرضية البحث والتي تعني " بوجود فجوة بين واقع النظام المحاسبي الموحد والقاعدة المحاسبية رقم (14) وبين معايير الابلاغ الملالي الدولية في ما يتعلق بالمحاسبة عن الاستثمارات المالية".

النتائج والتوصيات: النتـائج:

في ضوء ما تم مناقشته في هذا البحث فقد تو صل الباحثان الم بجموعة من الاستنتاجات و التي هي ما يأتي: 1 - وجود فجوة واسعة بين واقع النظام المحاسبي الموحد المطبق في العراق وبين متطلبات معايي الابلاغ الملالي الدولية مثلا ان الاستثارات المالية تصنف بموجب النظام المحاسبي الموحد حسب القطاعات وبالتصنيف التقليدي. 
2- عدم تو افق القاعدة المحاسبية العراقية رقم (14 ) "المحاسبة عن الاستثمارات" مع متطلبات معايير الابلاغ الملالي الدولية من حيث تسمية الاستثمارات المالية وتعريفها حيث وردت في القاعدة المحاسبية العراقية رقم (14 ) تحت مسمى الاستثمارات المالية والتي شملت جميع الاصول، بينما وردت في معايير الابلاغ الملالي الدولية بمسمى الادوات المالية والتي تعني اي عقد ينشأ على الاصول المالية للمنشاة والالنزامات المالية على المنشاة وحقوق المساهمين لمنشات اخرى. 3 - تبين نتائج البحث ايضا اختلاف بين القاعدة المحاسبية رقم (14 ) وبين معايير الابلاغ المالي الدولية من حيث نطاق التطبيق، حيث تطبق الاولى في جميع المنشات دون استثاء، بينما يطبق معيار الابلاغ الملالي رقم (9) من قبل جميع المنشات وعلى جميع الادوات المالية وباستثناءات محددة. 4 - كما تبين نتائج البحث عدم توافق القاعدة المحاسبية رقم (14) مع معايير الابلاغ الملالي من حيث تصنيف الاستثمارات المالية واثباتها، حيث صنفت الاولى المى استثمارات قصيرة الاجل وتدرج بسعر الكلفة او السوق ايهلا اقل في تاريخ الميزانية والمى استثارات طويلة الاجل وتدرج بسعر الكلفة، بينما صنفت وفق معايير الابلاغ الملالي المى عدت تصنيفات منها تقليدية تشمل ادوات مالية قصيرة ومتوسطة وطويلة الاجل واخرى الاكثر شيوعا وهي استثمارات مسوكة حتى تاريخ الاستحقاق وتدرج على اساس التكلفة المستهلكة واستثمارات ممسوكة حتى تاريخ البيع واستثمارات ممسوكة لغرض المتاجرة والتي تقاس كل منها بالقيمة العادلة. 5-وجود اختلاف محدود من حيث الافصاح عن الاستثمارات المالية في ضوء القاعدة المحاسبية رقم (14) ومعايير الابلاغ الملالي الدولية، وفق القاعدة المحاسبية العراقية تفصح الشركات في الميزانية عن الاستثمارات طويلة الاجل ضمن الموجودات الثابتة، والاستثمارات قصيرة الاجل ضمن الموجودات المتداولة والافصاح في تقرير الادارة عن القيمة السوقية للاستثمارات القابلة للتداول، بينما تفصح الشركات وفق معايير الابلاغ الملالي عن القيم الفردية للأنواع الثلاثة من الاستثمارات في الميزانية او في الملاحظات الواردة فيها ويجب التقرير عن الاستثارات بغرض المتاجرة بأجمالي قيمتها العادلة ضمن 
الاصول المتداولة بينا تعرض الاستثمارات الممسوكة حتى الاستحقاق والمتاحة للبيع كأصول متداولة او غير متداولة بناءا على الظروف المحيطة.

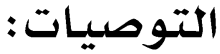

في ضوء ما تم تناوله في هذا البحث فقد تو صل الباحثان الى مجموعة من التوصيات و التي هي ما يأتي:

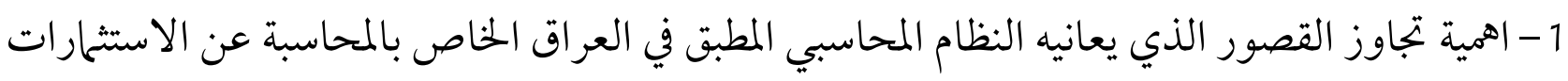
المالية والمتعلق بتصنيف الاستثمارات المالية واثباتها والمعالجات المحاسبية والافصاح عنها في القوائم المالية.

2- ضرورة اصدار قاعدة محاسبية خختصة بالاستثلارات المالية وقاعدة اخرى تتضمن الاستثمارات في المنشات الزميلة والمشروعات المشتركة وقاعدة اخرى تتعلق بالعقارات الاستثمرية ووفق معايير الابلاغ الملاي الدولية وبشكل منفصل من اجل تجاوز الدمج في تصنيفها وقياسها والمعالجات المحاسبية لكل منها والافصاح عنها في القو ائم المالية.

3 - اهمية تو حيد المصطلحات الواردة في القاعدة المحاسبية رقم (14 ) والنظام المحاسبي الموحد مع معايير الابلاغ المالي الدولية وتوضيحا بالشكل الذي يجعلها اكثر شمولا لجميع الاصول المالية والالتزامات المالية وحقوق المساهمين وتصنيفها وفق اسس علمية واضحة بلا يتوافق مع معايير الابلاغ المالي

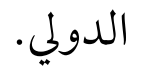

4- ضرورة تصنيف الاستثلمات المالية التقليدية (استثلارات طويلة الاجل، واستثمارات قصيرة الاجل) الواردة في النظام المحاسبي الموحد والقاعدة المحاسبية رقم (14) الى استثلارات مسوكة حتى تاريخ الاستحقاق واستثلمارات مالية مسوكة حتى تاريخ البيع واستثلمارات مسوكة لغرض المتاجرة وفقا لما جاء في معايير الابلاغ المالي الدولية. 
5- اهمية الافصاح عن الاستثمارات المالية في التقارير المالية وفقا للقيم الفردية لكل نوع من انواع الاستثلارات في الميزانية او الملاحظات الواردة فيها، من اجل التسهيل على مستخدمي البيانات المالية للتأكد من التدفقات النقدية الناتجة عن كل نوع من انواع الاستثمارات.

\section{المراجع والمصادر

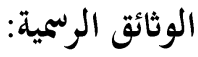

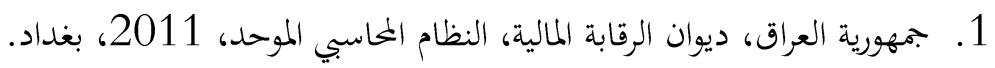

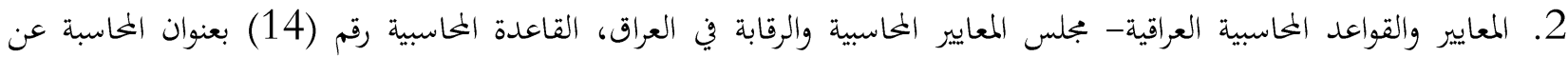

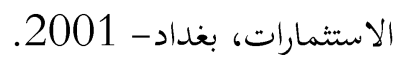

3. الجممع العربي للمحاسبين القانونيين، 2007، معايير المحاسة والابلاغ المالي الدولية الصادرة عن مجلس معايير المحاسبة الدولية،

$$
\text { منشور الجمع العربي للمحاسبين القانونيين. }
$$

4. المعايير الدولية لاعداد التقارير المالية، 2016، معيار الابلاغ المالي الدولي رقم (9)، ترجمة الهيئة السعودية للمحاسبين القانونيين.

5. ابو نصار وحميدات، محمد، جمعة، 2016، معايير المحاسبة والابلاغ المالي الدولية، دار وائل للطباعة والنشر، عمان- الاردن .

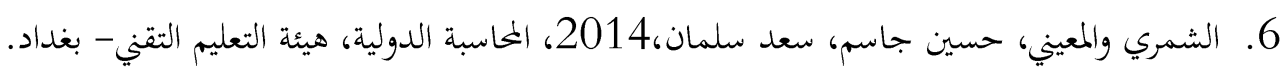
7. 7 عبد القادر، محسن بابقي، 2013، المحاسبة الدولية، جامعة العلوم والتكنلوجيا ، صنعاء.

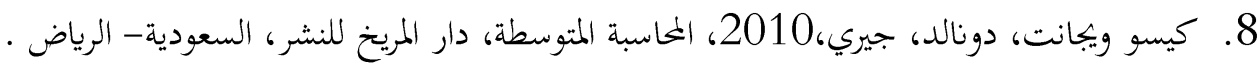
9. توفيق، اشرف مصطفى، 2008، كيف تتعلم استثمار الاموال في البورصة وصناديق الاستمار واسواق راس المال، دار ايتراك للطباعة والنشر، مصر. اطاريح الدكتوراه ورسائل الماجستير:

10. محمد، حاكم محسن، 2008، تحليل البيئة الاستثمارية للاستمار المالي والاجنبي في العراق، جامعة كربالا، كلية الادارة والاقتصاد.

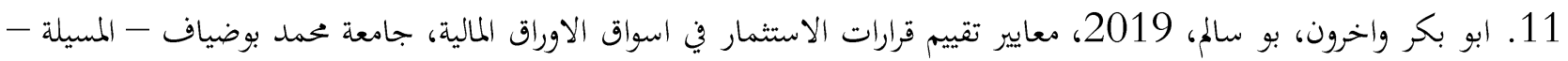

$$
\text { معهد العلوم الاقتصادية. }
$$

12. نصار واخرون، محمد محمود، 2018، خخاطر الاستثمار في الوراق المالية، كلية الدراسات العليا في الادارة .

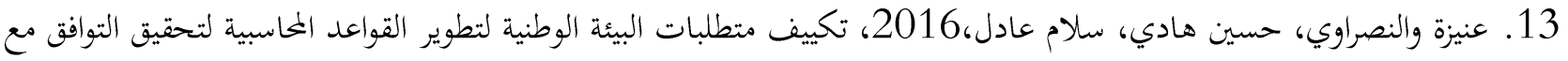

$$
\text { المعايير المحاسبية الدولية، كلية الادارة والاقتصاد- جامعة القادسية. }
$$

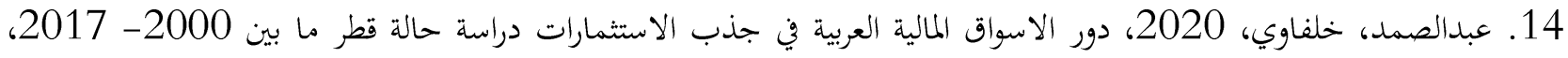

$$
\text { جامعة زيان عاشور الجلفة- قسم العلوم الاقتصادية. }
$$


15. وسام، شالور، 2011، المعالجة المحاسبية للادوات المالية في ظل معايير المحاسة الدولية، جامعة فرحان عباس سطيف- قسم العلوم

$$
\text { التجارية. }
$$

16. معروف والعتابي، احمد صلاح، 2019، اثر تداول اسهم غير العراقيين في الاستثمار المالية، كلية المأمون الجامعة، بجلة دراسات

$$
\text { محاسبية ومالية ،العدد 47، بحلد 14. 14. }
$$

17. نجم وناصر، بان توفيق، 2011، التقويم الخاسبي للاستثمارات ومدى الملائمة مع المعايير الخاسبية في دول مجلس التعاون الخليجي،

$$
\text { جامعة البصرة، مجلة الاقتصاد الخليجي، العدد } 19 .
$$

18. اممد وممود، وريا برهان، 2009، امكانية تطوير النظام المحاسبي الموحد المطبق في المصارف العراقية في ضوء معايير الماسبة

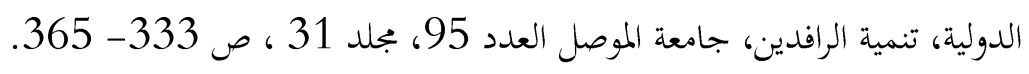

19. خليل، حوراء احسان، 2018، دور معايير المحاسة الدولية في الافصاح عن الادوات المالية المشتقة في القوائم المالية، الميئة العراقية

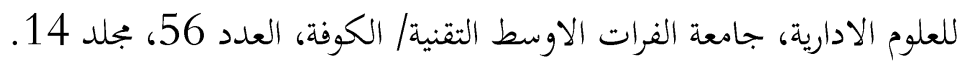

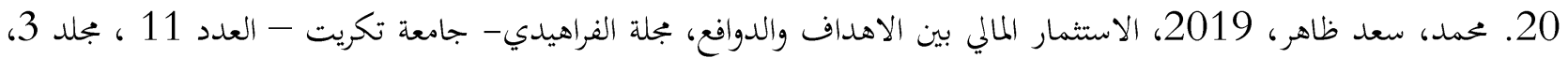

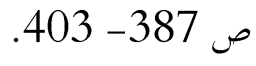

21. سوق العراق للاوراق المالية - التقرير السنوي، دليل الشركات: www.bsairaq.net

$$
\text { المصادر الاجنبية: - n }
$$

22. Kisso ، Donald، Others، 2015، Intermediate Accounting 